\title{
Utilizing information technology to improve transition of care from hospital to home
}

\author{
Dorothy G. Andrew ,' Susan E. Puls, Kerrie S. Guerrero \\ Houston Methodist Hospital, Houston, United States
}

Received: July 14, 2015

Accepted: October 8, 2015

Online Published: February 16, 2016

DOI: $10.5430 /$ jnep.v6n6p61

URL: http://dx.doi.org/10.5430/jnep.v6n6p61

\begin{abstract}
Background and objective: Failure to appropriately plan for a safe and effective transition to the next level of care leads to a greater use of hospital and emergency services, often measured by rates of readmission. A large academic medical center located in Houston, Texas, USA consistently achieves an overall University Health System Consortium (UHC) ranking for most benchmarks in the top decile (90th percentile) except for 30-day all-cause readmission rate, which ranks in the bottom decile. The objective of the study was to implement changes in Midas+, the system used by Houston Methodist Hospital for quality and case management activities, select a formal transition of care plan and implement the process on a pilot unit to reduce the 30-day readmission rate and improve the discharge planning process.

Methods: Setting: Cardiovascular Intermediate Care Unit (CVIMU), a 30-bed cardiovascular surgery unit within an academic medical center in Houston Texas. The project intervention included the addition of a readmission risk screen in the Hospital Case Management (HCM) and intervention screens based on the Coleman Model in the Community Case Management (CCM) module of Midas+. The clinical improvement involved three components spanning from hospital to home: (1) Screening patients for readmission risk upon admission and assigning those identified as high-risk for readmission (with a planned discharge to home) to a Transition Coach, (2) A visit by the Transition Coach during the patient's hospital stay to assess the patient and provide coaching, and (3) Providing five follow-up phone calls from the Transition Coach post-discharge.

Results: The system changes in Midas+ were implemented and were effective in tracking the interventions. Of the 258 patients admitted from July through August 2014, 226 or $87.5 \%$ of the patients were screened using the readmission risk assessment tool. Of the patients' screened, 49 were considered high risk with 26 discharged home, 22 or $45.8 \%$ discharged to another level of care and one patient expired. During the pilot, of 19 patients were followed by a transition coach only one patient readmitted to the hospital.

Conclusions: The project demonstrated that utilization of a computer system to record the readmission risk screen, track the assessment of the pillars (medication management, continued care, red flags to report, and personal health record) over the six time intervals of the pilot transition program was effective in tracking the intervention. The data collected through information technology was easily retrieved for tracking progress and evaluation. The outcome of this pilot has shown that a well-defined transition of care program may decrease the 30-day readmission rate.
\end{abstract}

Key Words: Transition of care, Discharge planning, Information technology, Reducing readmission

\section{INTRODUCTION}

An unplanned readmission to the hospital within 30 days of discharge is often seen as a failure by the healthcare team to appropriately plan for a safe and effective transition to the next level of care. ${ }^{[1]}$ Older patients, especially those with complex care needs, are particularly vulnerable to experiencing serious quality problems transitioning between different health care settings. ${ }^{[2]}$ Studies consistently have

*Correspondence: Dorothy G. Andrew; Email: dgandrew3@ gmail.com; Address: Houston Methodist Hospital, Houston, United States. 
shown that patients are often unprepared for assuming selfmanagement of their care as they transition to the next care setting. ${ }^{[3]}$ Common reasons cited were that patients often received conflicting advice regarding illness management, had difficulty accessing health care practitioners who were knowledgeable about their plan of care, and also had minimal input into their own plan of care. A coordinated discharge or transition plan has been demonstrated to be effective in the reducing readmissions and the burden placed on the healthcare system. ${ }^{[3]}$

At the time of hospital discharge, many patients are at increased risk from the combination of shorter stays, increased severity of illness and more complex discharge plans. ${ }^{[4]}$ The impact of health literacy also contributes to a lack of understanding and adherence to the medical discharge plan. ${ }^{[5]}$ In addition, handoffs in care between providers are often a weakness increasing the potential for errors and unsafe care. $^{[4]}$

\subsection{Background knowledge}

The cost to provide healthcare is a significant burden on the federal budget. The Congressional Budget Office projects Medicare costs to increase from $\$ 622$ billion in 2015 to $\$ 1.2$ trillion in 2025. ${ }^{[6]}$ One area under focus to balance high quality and cost effective care is the reduction in the number of patients readmitted to the hospital within 30 days of discharge. A coordinated discharge or transition plan has been demonstrated to be effective in the reducing readmissions and the burden placed on the healthcare system. ${ }^{[3]}$

The Centers for Medicare and Medicaid Services (CMS) have developed a strategy to improve the quality of care provided to the Medicare population and reduce health care costs by shifting to improving payment for high quality. As Medicare has shifted to a pay for performance (P4P) strategy in 2012, a readmission rate higher than the national average for heart failure, pneumonia, or acute myocardial infarction resulted in a penalty. Starting in federal fiscal year 2015, this penalty is up to $3 \%$ of all Medicare inpatient reimbursement received by the hospital. ${ }^{[7]}$ From 2007 to 2011, the readmission rate for Medicare patients remained around 19\%. ${ }^{[8]}$ The readmission rate decreased in calendar year 2012 to $18.4 \%{ }^{[8]}$ Hospitals and professional societies have been developing and implementing programs in an attempt to reduce readmissions.

Hospitals and health systems are facing two important transitions with the move toward population management and value-based purchasing. Organizations will need to begin creating care delivery innovations in order to achieve and sustain new quality benchmarks in terms of episodes of care and care management. They must also be preparing for changing payment models that shift risk onto providers. ${ }^{[9]}$

A key element to care transformation efforts is the establishment of a comprehensive care management infrastructure. Initially, organizations focus on a targeted population, such as high-risk patients. High-risk patients represent disproportionate amounts of health care spending. According to a report by the Health Care Advisory Board (2012), the top $12 \%$ of Medicare beneficiaries with multiple chronic illnesses account for $43 \%$ of total dollars spent. Lack of coordination results in high-cost health care utilization, which may result in an avoidable readmission or visit to an Emergency Department for care. These unplanned Emergency Department visits and hospital readmissions cost Medicare $\$ 26.4$ billion a year. ${ }^{[10]}$ Focusing efforts on high-risk patients allows organizations to allocate limited resources in such a way as to maximize outcomes and achieve specific care management objectives. ${ }^{[9]}$

\subsection{Literature review}

The project team conducted a literature review to determine current evidence available on strategies to reduce 30-day readmissions for the hospitalized patient. The search included PubMed and Medline (OVID) for articles and studies published between these databases' inception and March 2013. Medical Subject Headings (MeSH) and text terms were used, which included home care services, continuity of patient care, patient discharge, patient-centered care, health planning, patient readmission, and adult. The reference lists in the studies were also reviewed for potential additional studies missed in the database search. The initial search did not include articles written by Eric A. Coleman, MD, who is well known for his research and work in care transitions, therefore a second database search was conducted, adding the search term "Coleman" in order to retrieve his articles. Selection criteria included studies written in English in which a transition of care intervention, including a nursing component, was implemented before, during, or after hospitalization to adult patients hospitalized in an acute care setting who were being discharged to home. The outcome measure of interest was readmissions. Also considered were studies in which tools were developed or tested to identify patients at high risk for difficult transition. ${ }^{[11]}$

Multiple articles described transitional care as is a set of interventions designed to coordinate the care during the movement between healthcare settings. ${ }^{[1-4]}$ The process is intentional, clearly defined with expectations and accountability, focusing on the patient and caregiver needs. ${ }^{[12]}$ During the hospitalization, the intervention is with a responsible expert or team who assesses needs and develops the plan for 
care required post-discharge. Post-discharge interventions include home visits and telephone follow-up calls to reinforce teaching, as well as to provide support to the patient and caregiver. ${ }^{[2]}$

Another area of literature reviewed was related to the use of computer systems to support clinical quality programs. The Institute of Medicine (IOM) supports the use of clinical Information Technology (IT) systems to improve care that are designed to make it easy to do the right thing. ${ }^{[12]}$ In the IOM (2012) report on Health IT and patient safety, a strong case is made for how to improve providers' performance, enhance communication with patients and leading to an improvement in patient safety while reducing costs. ${ }^{[13]}$

The focus of this article is to describe a pilot patient education program utilizing the current Midas+ Case Management module to facilitate tracking and monitoring implementation of the transition process. The system improvements included screens for Readmission Risk Assessment and Transitional Care Coaching screens to track process, coaching actions, and patient response. A work list in MIDAS was assigned to the Transition Care Coaching Pilot. Once the screens in the MIDAS system were modified and updated to the live system, they were used to identify patients at high risk and track progress in the patient/caregiver knowledge for self-care for 30 days post discharge.

\section{MethodS}

Midas+ is a quality management product for the acute care setting providing integrated solutions addressing strategic performance management. The Midas+ product line was first developed in 1987 by James E. Peebles, a former hospital information systems executive and co-founder of Sunquest Information Systems, Inc., and Christopher J. Heller, MD, a surgeon active in hospital quality management. ${ }^{[14]}$

Midas+ is he computer system used at Houston Methodist Hospital (HMH) to document the quality and case management programs and was utilized for this project. The base system includes capability for programming a readmission risk screen in the Hospital Case Management (HCM) module and intervention screens based on the Coleman Care Transition Intervention Model in the Community Case Management (CCM) module. ${ }^{[3]}$ Working with the IT team, the program changes included:

(1) Computer program changes including:

A. Activation of the Readmission Risk Screen in the HCM module

B. Implementation of the Transition Care Coaching (TCC) Work List for cases identified as high risk

C. Addition of TCC Assessment Screens to docu- ment patient/caregiver knowledge related to medication management, personal health record, continuing care and how to identify red flags to report to the physician. The system has capacity to track six contacts with the patient/caregiver - the in hospital assessment, initial discharge phone call, and four weekly phone calls.

D. Addition of dictionaries to track coaching actions for each pillar. Examples of actions include reminders to develop a medication reminder, questions or documents to bring to physician visits and red flags to report such as weight gain, fever, swelling, increased pain, swelling or drainage.

(2) System generated reports to track the progress and outcome of identification assessment, coaching and follow-up of high risk patients and caregivers were developed and added to the report menu.

The project team consisted of three doctor of nursing practice students with one student completing the risk assessments and two students providing the discharge education and support. The intervention began shortly after admission to the hospital by completion of a readmission risk assessment. A screening tool developed by the Society of Hospital Medicine as part of the Better Outcomes by Optimizing Safe Transitions (BOOST) program formed the basis of the screening tool used for this project. ${ }^{[15]}$ A panel of national experts on transitional care, chaired by Dr. Eric Coleman and Dr. Mark Williams, developed a tool identifying eight problem areas (8Ps) that increase the risk of readmission including prior readmission, problem medications or polypharmacy (> 10 medications), problem diagnoses, poor health literacy, psychosocial issues, poor family/community support, problem with finances, and problem with mobility. ${ }^{[16]}$ The screening tool for the project separated polypharmacy and problem medications resulting in nine problem areas or 9Ps (see Figure 1).

The HCM module contains a screen that includes a readmission risk screen with the ability to send positive screens to a work list. The screen content is based on nine problem areas that increase the risk of readmission. Patients admitted to the pilot unit were screened early in their stay for readmission risk. The process include review of the medical history and physical, the nursing admission assessment, and medication reconciliation documents.

Patients at high risk for readmission were assigned to a transition care coaching worklist. The Transition Coach (TC) interviewed the patient, completed an assessment, and developed a plan. In the Midas+ system, the CCM module contains an assessment tool to evaluate patient/caregiver knowledge in 
the four pillars and the ability to track the activities of the coach over time (see Figures 2 and 3). The results of the initial assessment, first phone call within 48 hours of discharge and the four weekly follow-up calls were recorded in the CCM module in the Midas+ system. The patient/caregiver knowledge in each pillar received a score as 0 or 1 . Coaching actions completed during the assessment were recorded in the system.

\section{Statistical analysis}

A statistician was consulted to perform a statistical analysis of the project data. Data are presented as means (standard deviation) for continuous variables and numbers (percentage) are reported for categorical variables. Comparison of categorical data was done by Fischer's exact test. A one-way ANOVA was used to test for significant differences among scores during all timepoints. All analyses were performed with STATA version 13.0 (StataCorp, College Station, TX). Statistical significance is defined as a two-tailed $p<.05$ for all tests.

\section{Results}

Computer program. The Midas+ system changes involved the HCM and CCM modules. In the HCM module, the Readmission Risk screen was activated for Dunn 9 West patients and the project team was granted access (see Figure 1). Find- ings from the screening were recorded by the number of problem areas identified-none, one or two, three, four or more, and 30-day readmission. From this screen, patients with four or more problem areas or were currently a 30-day readmission were considered high risk for readmission and mapped to the Transitional Care Coaching work list in the CCM module. The TCC assessment and coaching screens were programmed with the four pillars for assessment (medication management, follow-up with a primary care physician, the use of a personal health record, and red flags to report) with a drop down box for documentation of the coaching interventions, a comment section and an area to record the time spent on the activity (see Figures 2 and 3). The TC completed an assessment for each time interval, selecting one of six time categories (inpatient, 48 hours post discharge, and four weekly calls) from a drop down box. The system successfully tracked all patients through the process. Reports from the system tracked the number of screenings by category.

The results for discharge location based on reassessment risk are displayed in Table 1. The data show that there is a significant relationship between the risk score and discharge location. Patients with a higher risk score were discharged to another level of care more frequently than patients with a lower risk score. One key finding was the number of patients not discharged to home but to another level of care.

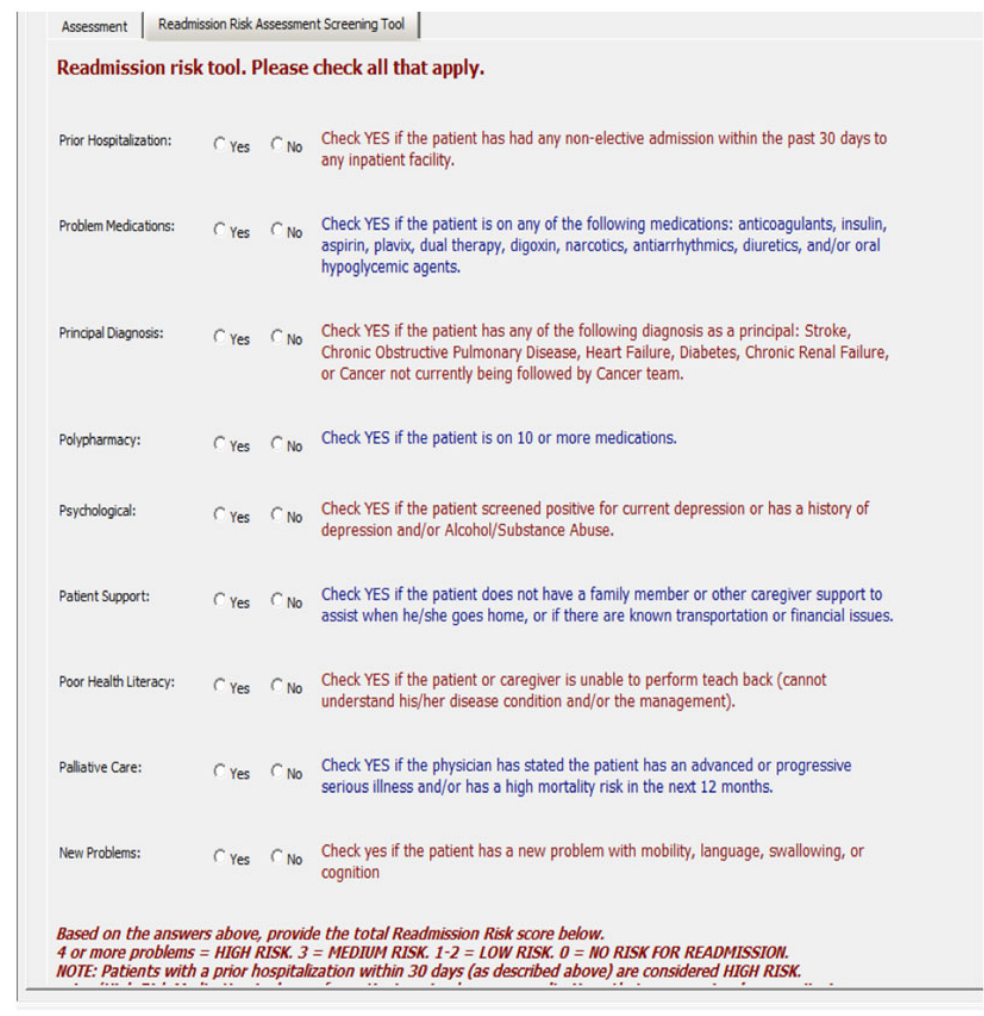

Figure 1. Reassessment risk tool 


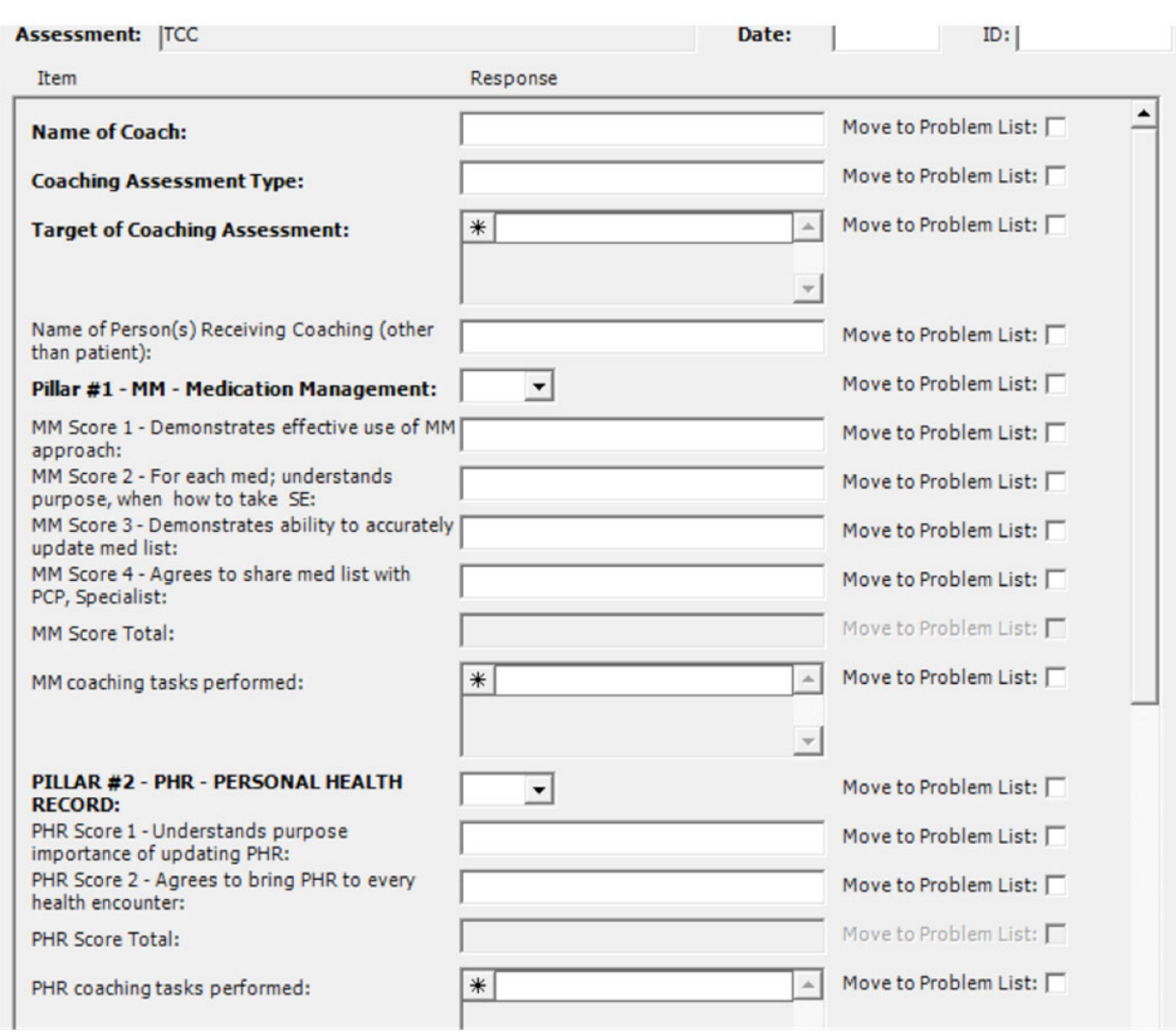

Figure 2. Transitional care coach assessment tool Part 1

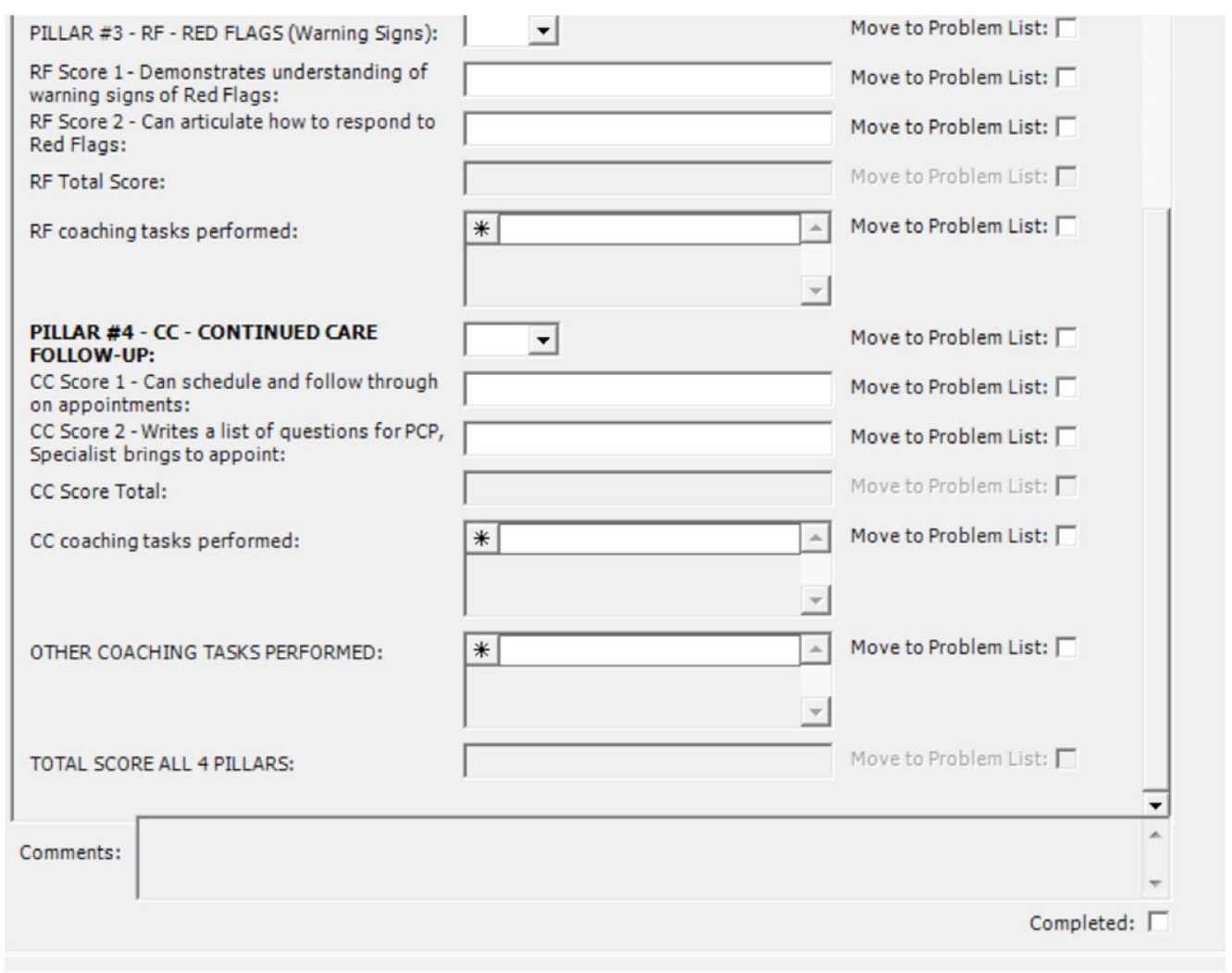

Figure 3. Transitional care coach assessment tool Part 2 
Of the 222 screened patients discharged, 26 were admitted hospital, and nine to an inpatient rehabilitation facility. This to a skilled nursing facility, 10 to a long term acute care represented $20.3 \%$ of screened patients who were discharged.

Table 1. Discharge destination based on readmission risk score $(p=.001)$

\begin{tabular}{|c|c|c|c|c|c|}
\hline Readmission Destination & High Risk $(\geq 4)$ or Readmission & Moderate Risk (3) & Low Risk (1-2) & No Risk (0) & Total \\
\hline \multirow{3}{*}{ Expired } & 1 & 1 & 2 & 0 & 4 \\
\hline & 25.00 & 25.00 & 50.00 & 0.00 & 100.00 \\
\hline & 2.04 & 2.38 & 1.79 & 0.00 & 1.77 \\
\hline \multirow{3}{*}{$\begin{array}{l}\text { Home (With or Without } \\
\text { HHC) }\end{array}$} & 26 & 33 & 97 & 21 & 177 \\
\hline & 14.69 & 18.64 & 54.80 & 11.86 & 100.00 \\
\hline & 53.06 & 78.57 & 86.61 & 91.30 & 78.32 \\
\hline \multirow{3}{*}{$\begin{array}{l}\text { Long Term Acute Care } \\
\underline{\text { Hospital }}\end{array}$} & 6 & 1 & 1 & 1 & 9 \\
\hline & 66.67 & 11.11 & 11.11 & 11.11 & 100.00 \\
\hline & 12.24 & 2.38 & 0.89 & 4.35 & 3.98 \\
\hline \multirow{3}{*}{ Acute Inpatient Rehab } & 4 & 1 & 5 & 0 & 10 \\
\hline & 40.00 & 10.00 & 50.00 & 0.00 & 100.00 \\
\hline & 8.16 & 2.38 & 4.46 & 0.00 & 4.42 \\
\hline \multirow{3}{*}{ Skilled Nursing Facility } & 12 & 6 & 7 & 1 & 26 \\
\hline & 46.15 & 23.08 & 26.92 & 3.85 & 100.00 \\
\hline & 24.49 & 14.29 & 6.25 & 4.35 & 11.50 \\
\hline \multirow{3}{*}{ Total } & 49 & 42 & 112 & 23 & 226 \\
\hline & 21.68 & 18.58 & 49.56 & 10.18 & 100.00 \\
\hline & 100.00 & 100.00 & 100.00 & 100.00 & 100.00 \\
\hline
\end{tabular}

Note. For each readmission destination, Row 1 = number of patients, Row 2 = row \%, and Row $3=$ column $\%$.

Of the 49 high-risk patients, 22 patients were excluded and did not receive any transition of care intervention. Reasons for exclusion included patients with a planned discharge to another facility, patients missed by the project team, and one patient who expired. Eight patients received an inpatient baseline assessment only and did not receive the postdischarge portion of the intervention. This was primarily due to the planned discharge destination changing, although one patient was dropped due to being non-English speaking. The remaining 19 patients received both inpatient and post-discharge components of the transition of care intervention described earlier in this paper. The breakdown of the high-risk patients can be seen in Table 2 .

The project team reviewed the readmission rate information for just those patients screened as part of this pilot. Of the 226 patients screened 28 , or $12.39 \%$ were readmitted. Breaking this down further, of the 28 patients readmitted, only one came from the group of 19 patients who received the transition of care intervention; thus the readmission rate for the patients receiving both inpatient and post-discharge components of the intervention was 5.26\%. The remaining 27 patients who were readmitted did not receive the intervention, which resulted in a readmission rate of $13.04 \%$ for screened patients not receiving the intervention. Although the CVIMU 30-day all-cause readmission rate did not improve, the readmission rate for patients identified as high-risk for readmission receiving the transition of care intervention was lowest between the two groups (those receiving the intervention and those not receiving the intervention) (see Table $3)$.

Another area of interest to the project team was whether or not a patient's readmission risk score predicted the likelihood of readmission. Although the project did not demonstrate statistical significance between a patient's readmission risk score and whether or not the patient was readmitted ( $p=$ .482), the readmission rate for patients identified as high-risk for readmission was the highest of all groups (see Table 4).

Table 2. Breakdown of 49 high-risk patients

\begin{tabular}{|c|c|c|}
\hline $\begin{array}{l}\text { Received Both Inpatient and Post-discharge } \\
\text { Components of Intervention }\end{array}$ & Inpatient Baseline TCC Assessment Only & No Intervention \\
\hline $\begin{array}{l}\text { - } 19 \text { patients discharged home (with or without } \\
\text { HHC) }\end{array}$ & $\begin{array}{l}\text { - } 5 \text { patients with change of discharge destination from } \\
\text { home to LTAC/SNF/Rehab } \\
\text { - } 1 \text { patient discontinued (non-English speaking) } \\
\text { - } 2 \text { patients missed by TC }\end{array}$ & $\begin{array}{l}\text { - } 17 \text { patients with planned discharge } \\
\text { to LTAC/SNF/Rehab } \\
\text { - } 4 \text { patients missed by TC } \\
\text { - } 1 \text { patient expired }\end{array}$ \\
\hline
\end{tabular}

Note. HHC (Home Health Care); LTAC (Long Term Acute Care); SNF (Skilled Nursing Facility); Rehab (Rehabilitation facility); TC (Transition Coach). 
Table 3. Readmission rate results

\begin{tabular}{ll}
\hline Outcome Measure & Results \\
\hline $\begin{array}{l}\text { Pilot unit 30-day all-cause readmission rate within } 3 \\
\text { months of implementing PIP. }\end{array}$ & $\begin{array}{l}2^{\text {nd }} \text { Quarter 2014-9.24\% (Baseline) } \\
3^{\text {rd }} \text { Quarter 2014-11.44\%* }\end{array}$ \\
$\begin{array}{l}\text { Percentage of patients screened for readmission risk that } \\
\text { were readmitted. }\end{array}$ & $12.39 \%$ of patients screened for readmission risk were readmitted. \\
$\begin{array}{l}\text { Percentage of patients who did not receive the } \\
\text { intervention who were readmitted. }\end{array}$ & $13.04 \%$ of patients who did not receive the intervention were readmitted. \\
$\begin{array}{l}\text { Percentage of patients receiving the intervention who } \\
\text { were readmitted. }\end{array}$ & $5.26 \%$ of patients receiving the intervention were readmitted. \\
$\begin{array}{l}\text { Percentage of high-risk patients who did not receive the } \\
\text { intervention who were readmitted. }\end{array}$ & $26.67 \%$ of high-risk patients who did not receive the intervention who were readmitted. \\
$\begin{array}{l}\text { Percentage of moderate-risk patients readmitted. } \\
\text { Percentage of low to no risk patients readmitted. }\end{array}$ & $11.90 \%$ of moderate-risk patients readmitted. \\
\hline
\end{tabular}

*No improvement noted.

Table 4. Readmission status based on readmission risk score $(p=.482)$

\begin{tabular}{llllll}
\hline Readmission Status & $\begin{array}{l}\text { High Risk (score } \geq \text { 4) } \\
\text { Readmission }\end{array}$ & $\begin{array}{l}\text { Moderate Risk } \\
\text { (score 3) }\end{array}$ & $\begin{array}{l}\text { Low Risk } \\
\text { (score 1-2) }\end{array}$ & $\begin{array}{l}\text { No Risk } \\
\text { (score 0) }\end{array}$ & Total \\
\hline \multirow{2}{*}{ No } & 39 & 36 & 99 & 20 & 194 \\
& 20.10 & 18.56 & 51.03 & 10.31 & 100.00 \\
Yes & 81.25 & 87.80 & 90.00 & 86.96 & 87.39 \\
& 9 & 5 & 11 & 3 & 28 \\
Total & 32.14 & 17.86 & 39.29 & 10.71 & 100.00 \\
& 18.75 & 12.20 & 10.00 & 13.04 & 12.61 \\
& 48 & 41 & 110 & 23 & $222^{*}$ \\
\end{tabular}

Note. Value in first row for each Readmission Status category denotes frequency, second row denotes row percentage, and third row denotes column percentage. $* 4$ of the 226 patients screened expired and were not included in this readmission status analysis.

\section{TCC assessment scores}

In addition to a numerical score, a patient's TCC Assessment guided the coaching efforts on any of the four area(s) that scored low. With weekly TCC re-assessment and coaching, the project team was interested in evaluating whether or not these efforts improved the TCC Assessment scores. For the high-risk patients receiving the intervention, a statistically significant $(p=.0000)$ increase was demonstrated in the overall total mean TCC Assessment score from the first inpatient baseline assessment to the final assessment completed post-discharge (see Table 5).

Statistically significant increases were also noted in the mean scores for each of the four target areas (medication management, personal health record, red flags, and continuing care), the detail of which is shown in Table 6.

\section{Discussion}

The utilization of a computer system to record the readmission risk screen, track the assessment of the pillars (medication management, continued care, red flags to report, and personal health record) over the six time intervals of the pilot transition program was effective. Midas+ was easy to use to screen for readmission risk and high risk patients were automatically transferred to the work list. The coaching Published by Sciedu Press screens were available and all entries recorded were retrievable. Since the Midas+ system is used at all six Houston Methodist facilities, information identifying readmissions to any Houston Methodist facility or emergency department visit was readily available. For analytical purposes, the data from Midas+ was exportable in excel spreadsheets for statistical evaluation. The results of the multiple coaching interventions assessing patient/caregiver knowledge allowed tracking results over time. In addition, the total time required for the coaching interventions was recorded. This result allows an evaluation of the feasibility to spread the pilot to other areas of the facility.

Table 5. Summary of TOTAL Score* for All 4 Pillars $(p=$ .0000)

\begin{tabular}{lll}
\hline TCC Assessment Time & Mean & Std. Dev. \\
\hline Inpatient Baseline & 3.6522 & 2.5692 \\
24-48 hr. Post-discharge & 6.8889 & 2.7630 \\
1 wk. Post-discharge & 7.5882 & 2.2929 \\
2 wks. Post-discharge & 7.9412 & 2.4359 \\
3 wks. Post-discharge & 8.7222 & 1.9646 \\
4 wks. Post-discharge & 8.8462 & 1.7246 \\
4+ wks. Post-discharge & 5.0000 & 2.8284 \\
Total Mean Score & 6.9815 & 2.9765 \\
\hline
\end{tabular}

*Total possible points $=10$. 
Table 6. Mean scores for each target area

\begin{tabular}{|c|c|c|}
\hline \multicolumn{3}{|c|}{ Summary of MEDICATION MANAGEMENT Score* $(p=.0000)$} \\
\hline TCC Assessment Time & Mean & Std. Dev. \\
\hline Inpatient Baseline & 1.6087 & 1.3052 \\
\hline 24-48 hr. Post-discharge & 2.8889 & 1.4507 \\
\hline 1 wk. Post-discharge & 3.0588 & 1.1974 \\
\hline 2 wks. Post-discharge & 3.2353 & 1.2515 \\
\hline 3 wks. Post-discharge & 3.7222 & 0.7519 \\
\hline 4 wks. Post-discharge & 3.8462 & 0.5547 \\
\hline $4+$ wks. Post-discharge & 2.5000 & 2.1213 \\
\hline Total Mean Score & 2.9444 & 1.3796 \\
\hline \multicolumn{3}{|c|}{ Summary of PERSONAL HEALTH RECORD Score* $(p=.0205)$} \\
\hline TCC Assessment Time & Mean & Std. Dev. \\
\hline Inpatient Baseline & 0.6087 & 0.7223 \\
\hline 24-48 hr. Post-discharge & 1.0000 & 0.9701 \\
\hline 1 wk. Post-discharge & 1.1765 & 0.9510 \\
\hline 2 wks. Post-discharge & 1.2941 & 0.9196 \\
\hline 3 wks. Post-discharge & 1.3889 & 0.9164 \\
\hline 4 wks. Post-discharge & 1.6154 & 0.7679 \\
\hline $4+$ wks. Post-discharge & 0.5000 & 0.7071 \\
\hline Total Mean Score & 1.1204 & 0.9142 \\
\hline \multicolumn{3}{|c|}{ Summary of RED FLAGS Score* $(p=.0000)$} \\
\hline TCC Assessment Time & Mean & Std. Dev. \\
\hline Inpatient Baseline & 0.5217 & 0.7903 \\
\hline 24-48 hr. Post-discharge & 1.7222 & 0.6691 \\
\hline 1 wk. Post-discharge & 1.8235 & 0.5286 \\
\hline 2 wks. Post-discharge & 1.8235 & 0.5286 \\
\hline 3 wks. Post-discharge & 1.8889 & 0.4714 \\
\hline 4 wks. Post-discharge & 1.7692 & 0.5991 \\
\hline 4+ wks. Post-discharge & 1.0000 & 1.4142 \\
\hline Total Mean Score & 1.5185 & 0.8144 \\
\hline \multicolumn{3}{|c|}{ Summary of CONTINUED CARE Score* $(p=.0000)$} \\
\hline TCC Assessment Time & Mean & Std. Dev. \\
\hline Inpatient Baseline & 0.9130 & 0.5146 \\
\hline 24-48 hr. Post-discharge & 1.2778 & 0.5745 \\
\hline 1 wk. Post-discharge & 1.5294 & 0.5145 \\
\hline 2 wks. Post-discharge & 1.5882 & 0.5073 \\
\hline 3 wks. Post-discharge & 1.7222 & 0.4609 \\
\hline 4 wks. Post-discharge & 1.6154 & 0.5064 \\
\hline $4+$ wks. Post-discharge & 1.0000 & 0.0000 \\
\hline Total Mean Score & 1.3981 & 0.5791 \\
\hline
\end{tabular}

*Possible points $=2$

This pilot program focused on providing additional education and support for patients being discharged home. In the high risk readmission group, $45.8 \%$ of the patients were not discharged to home but to another facility. The highest readmission source was from patients discharged to another level of care, with discharges to the SNF having the highest readmission rate. Closer collaboration with the entities, sharing the identified problem areas may lead to the development of a coaching program to support the patient through the transition from hospital to skilled facility to home. While the entire program may not be required, additional education related to the problem area, and a follow-up call from the hospital to the patient, caregiver, and staff at the next level of care may be beneficial to reduce readmissions.
The risk screening tool may not be as effective on surgical patients where the surgical intervention may be the major factor in readmission risk, not just the history of medications or other diseases. Additional investigation needs to occur to determine a surgical readmission risk assessment tool. The impact of all the problem areas and impact on readmission need additional investigation and perhaps each area needs a more specific focus during the hospital stay with follow-up post discharge.

The Transition Coach was able to establish a relationship with the patient and their caregivers in order to explain the purpose of the program, and based on the inpatient assessment, conduct focused coaching. The information the Tran- 
sition Coach provided to the patient seemed to help increase the patients' understanding of healthcare needs and the readiness to play an active role in their care after discharge. In addition, the Transition Coach facilitated pulling together a multidisciplinary team consisting of a case manager, pharmacist, and social worker to address any patient concerns or answer any questions. During the inpatient stay, the Transition Coach took the time to build a rapport with the patient, which seemed to contribute to the ease with which the patients received the post-discharge phone calls.

Continuing with the post-discharge period, each encounter with the Transition Coach included a TCC re-assessment and coaching. These coaching efforts resulted in improved TCC Assessment scores, which were statistically significant (see Tables 5 and 6). Having the same Transition Coach followup each week was also important for continuity of care. For example, one patient, when called by the Transition Coach, articulated that he was struggling with adjusting his medications. He mentioned that he had "gained several pounds of water weight," and was experiencing low blood pressures to the point of feeling lightheaded and faint. The Transition Coach was able to refer the patient that same day to a pharmacist through the hospital's care navigator program. The pharmacist called the patient and provided assistance with managing his medications. The patient avoided a readmission, and when called by the Transition Coach the following week, was doing much better.

\section{Conclusions}

The purpose of this study was to evaluate whether the implementation of a formal transition of care program would decrease 30-day all cause readmissions and improve the discharge process. The steps included identification of a transition model, development of a computer program to screen for readmission risk, and tracking patients at high risk for readmission from assessment through coaching for 30 days post discharge. Coleman's Care Transition Intervention Model was chosen for this pilot as it is designed to support patients and their caregivers to achieve the knowledge and ability for self-care and management through the transitions of care from one level to another. It would appear that this approach would be easy to implement in most clinical settings due to its simplicity, relatively low cost to implement, and that it can be applied to a broad range of acute and chronic conditions.

The CMS pay-for-performance measures are placing hospital reimbursement at risk if readmission rates for the selected medical and surgical conditions are higher than national averages. Hospitals are looking for effective methods to reduce the 30-day readmission rates. The transition of care project team was able to implement a systematic transition of care plan supported by a computer system to assist patient/caregivers to obtain the knowledge and resources needed to successfully care for themselves after hospital discharge to reduce the risk of readmission. The outcome of this pilot has shown that a well-defined transition of care program may decrease the 30 -day readmission rate.

\section{ACKNOWLEDGEMENTS}

We would like to thank Sheldon Block and Debbie Bruce, Houston Methodist Hospital Information Technology, for their work on the MIDAS Program to support the project. Jaya Paranilam, Methodist Research Institute for assistance with the statistical analysis of the data.

\section{Conflicts of InTEREST Disclosure}

The authors declare that there is no conflict of interest.

\section{REFERENCES}

[1] Sminkey P, Naylor MD. Theory into practice: Transitional care model's success demonstrates that evidence alone isn't enough. Commission for Case Management Certification IssueBrief. 2015. Available from: http://ccmcertification.org/sites/defau lt/files/downloads/2015/CCMC-Jan2015-IB-WEB.pdf

[2] Naylor MD, McCauley KM. The effects of a discharge planning and home follow-up intervention on elders hospitalized with common medical and surgical cardiac conditions. J Cardiovasc Nurs. 1999; 14(1): 44-54. Available from: http://www.ncbi.nlm.nih .gov/pubmed/10533691 PMid:10533691 http://dx.doi.org /10.1097/00005082-199910000-00006

[3] Coleman EA, Parry C, Chalmers S, et al. The care transitions intervention: results of a randomized controlled trial. Arch Intern Med. 2006; 166(17): 1822-1828. http://dx.doi.org/10.1001/arc hinte.166.17.1822

[4] Chugh A, Williams MV, Grigsby J, et al. Better transitions: improving comprehension of discharge instructions. Front Health Serv Manage. 2009 Spring; 25(3): 11-32. PMid:19382514

[5] Mancini MJ, Heckman MG, Dawson NL. Functional health literacy and understanding your medications at discharge. Mayo Clinic Proceedings. 2008; 83(5): 554-558. http://dx.doi.org/10.4065 /83.5. 554

[6] Medicare Payment Advisory Commission (Medpac). Report to the Congress: Medicare payment policy. Washington (DC): Medpac; 2014. Available from: http://www.medpac.gov/documents/r eports/mar 14

[7] Centers for Medicare and Medicaid Services. Readmission reduction program. 2014. Available from: http://www.cms.gov/Medicare /Medicare-Fee-for-Service-Payment/AcuteInpatientP 
PS/Readmissions-Reduction-Program.html

[8] Gerhardt G, Yemane A, Hickman P, et al. Data shows reduction in Medicare hospital readmission rates during 2012. Medicare \& Medicaid Research Review. 2013; 3(2): E1-E12. Available from: http://www. cms .gov/mmrr/Downloads/MMRR2013_ 003_02_b01.pdf PMid:24753966 http://dx.doi.org/10.56 00/mmrr.003.02.b01

[9] Health Care Advisory Board. High-risk patient care management: Prioritizing high-value opportunities for managing total cost of care. The Advisory Board Company. 2012. Available from: http://www .advisory.com/Research/Health-Care-Advis ory-Board/Studies/2012/High-Risk-Patient-Care-Man agement?WT.mc_id=eMail|Daily+Briefing $\mid$ Study $|\mathrm{HCAB}| \mathrm{H}$ igh+Risk+Care+Management+B |Feb222013

[10] Dartmouth Atlas Project \& PerryUndem Research Communication. The revolving door: A report on U.S. hospital readmissions. Princeton, NJ: Robert Wood Johnson Foundation. 2013. Available from: http://www.rwjf.org/en/library/research/2013/ 02/the-revolving-door--a-report-on-u-s--hospita l-readmissions.html

[11] Puls SE, Guerrero KS, Andrew D. Facilitating safe patient transition of care: A qualitative systematic review. Journal of Nursing Educa- tion and Practice. 2014; 4(6): 37-52. http://dx.doi .org/10.54 30/jnep.v4n6p37

[12] Berner ES. Clinical decision support systems: State of the art. Rockville, Maryland: Agency for Health Research \& Quality. 2009. Available from: http://healthit.ahrq.gov/sites/default/ files/docs/page/09-0069-EF_1.pdf

[13] Institute of Medicine. Health IT and patient safety: Building safer systems for better care. Washington, D.C.: The National Academies Press. 2012. Available from: http://www. nap. edu/download.p hp?record_id $=13269$

[14] Midas + Solutions Website. 2015. Available from: https://www. midasplus .com/pages/AboutUs/AboutUs . aspx

[15] Hansen LO, Greenwald J, Budnitz T, et al. Project BOOST: Effectiveness of a multihospital effort to reduce rehospitalization. Journal of Hospital Medicine. 2013; 8(8): 421-427. PMid:23873709 http://dx.doi.org/10.1002/jhm. 2054

[16] Society of Hospital Medicine. Risk Assessment-8P, Project BOOST $\AA$ implementation toolkit-touch points: admission, during hospitalization, and discharge. 2015. Available from: http://www.hospitalmedicine.org/Web/Quality_Innov ation/Implementation_Toolkits/Project_BOOST/Web/Qu ality_Innovation/Implementation_Toolkit/Boost/BOOS T_Intervention/Tools/Risk_Assessment.aspx 